\title{
Evaluating the results of the Momguard noninvasive prenatal test
}

Hae-Jin Hu, Young-Jun Kwon, Mijin Oh, Jihun Kim, Dae-Yeon Cho, and Dong-Hee Seo*

LabGenomics Clinical Research Institute, LabGenomics, Seongnam, Korea

Purpose: To evaluate the performance of the Momguard noninvasive prenatal test by tracing the 'screen positive' results based on preliminary samples from Korean cohorts.

Materials and Methods: This preliminary study is based on data collected by the LabGenomics Clinical Laboratory (Seongnam, Korea) with informed consent. Only pregnant women who underwent both the Momguard test and karyotyping were included in this study. Momguard test results were compared with those of the karyotyping analysis.

Results: Among the 38 cases with 'screen positive' results by Momguard, 30 cases also had karyotyping results available. In three trisomy (T) 18 and three T13 cases, the Momguard results were concordant with the karyotyping results. For the T21 cases, except for one case belonging to the mid-risk zone, Momguard results from 23 out of 24 cases matched the karyotyping results.

Conclusion: Momguard is a highly reliable screening tool for detecting T13, T18, and T21 cases in independent Korean cohort samples.

Key words: Aneuploidy, Down syndrome, Trisomy 18, Trisomy 13 syndrome, Prenatal screening.

\section{Introduction}

Traditional approaches to identifying fetal chromosomal abnormalities, such as Down or Edwards syndrome, include maternal serum tests and fetal nuchal translucency (NT) assessments using ultrasound scanning [1]. Maternal serum tests include a double marker test measuring two markers of $\beta$-human chorionic gonadotropin ( $\mathrm{hCG}$ ) and pregnancyassociated plasma protein $\mathrm{A}$; a triple marker test measuring three markers of hCG, alpha-fetoprotein (AFP), and unconjugated estriol (uE3) in the second trimester; and a quadruple marker test measuring four markers of $h C G, A F P, u E 3$, and inhibin $A[2,3]$. Recently, an integrated screening test that combines the results from an NT ultrasound and maternal serum tests has been used for noninvasive prenatal test (NIPT) [4]. If a test result indicates a sample is 'screen positive', chorionic villus sampling (CVS) or amniocentesis is performed to confirm the result. However, current maternal serum screening tests have a relatively low detection rate (50-95\%) and high false-positive rate (5\%) [5]. CVS and amniocentesis carry the risk of fetal loss, since they are invasive procedures and may present additional emotional burdens for the mother.

The discovery of the existence of cell-free fetal DNA (cffDNA) in maternal plasma in 1997 by Lo et al. [6] led to a new era for NIPT. Within the last two decades, advanced technologies such as digital polymerase chain reaction or massively parallel

Received: 2 December 2015, Revised: 9 December 2015, Accepted: 14 December 2015, Published: 31 December 2015

${ }^{*}$ Corresponding author: Dong-Hee Seo, M.D.

LabGenomics Clinical Research Institute, LabGenomics, B-6F, 700 Daewangpangyo-ro, Bundang-gu, Seongnam 13488, Korea.

Tel: +82-31-628-0730, Fax: +82-31-628-0701, E-mail: seo2023@labgenomics.com

Conflict of interest: The authors of this publication is working in the LabGenomics which developed the products related to the research being reported.

(c) This is an open-access article distributed under the terms of the Creative Commons Attribution Non-Commercial License (http://creativecommons.org/licenses/by-nc/4.0/) which permits unrestricted non-commercial use, distribution, and reproduction in any medium, provided the original work is properly cited.

(c) Copyright 2015 by the Korean Society of Medical Genetics 
sequencing (MPS) have been used to identify fetal cffDNA in maternal blood, differentiate fetal DNA from maternal DNA, and detect fetal chromosomal abnormalities [7-12]. These NIPT methods are promising, in that they are safe and show good clinical performance with sensitivities up to $100 \%$ and specificities up to 99\% [13]. Currently, in the United States, Sequenom, Verinata Health, Ariosa Diagnostics, and Natera offer NIPT services $[14,15]$. In Korea, Momguard, an independent NIPT protocol, was developed using a random massively parallel shotgun sequencing method [16]. A large-scale blind evaluation of the assay is currently in progress in multiple Korean cohorts [16]. In this study, we preliminarily evaluated the performance of Momguard by analyzing 'screen positive' results in samples from the independent Korean cohort.

\section{Materials and Methods}

\section{Study design}

This preliminary study is based on data collected by LabGenomics Clinical Laboratory (Seongnam, Korea) in 2015. We obtained approval for the study from our institutional review board (2013-03) and included only the participants who gave written informed consent. This study included pregnant women who were reported as being at high risk for fetal anomalies based on the Momguard test and those who underwent an amniocentesis to confirm fetal karyotype.

\section{Sample collection}

Maternal blood samples were collected in Cell-Free DNA BCT tubes (Streck, Omaha, NE, USA) and delivered to the laboratory within $24 \mathrm{hr}$ of collection. To isolate plasma from the maternal blood, a two-step centrifugation process was applied, with centrifugation at $1,600 \times g$ for $10 \mathrm{~min}$ and then at $16,000 \times g$ for $10 \mathrm{~min}$ at $4^{\circ} \mathrm{C}$. In $1.5-\mathrm{mL}$ tubes labeled with distinct sample codes, the isolated plasma was distributed into 1-mL aliquots. Aliquots were frozen at $-80^{\circ} \mathrm{C}$ until they were analyzed.

\section{Cell-free DNA (cfDNA) extraction and sequencing}

To isolate cfDNA from $2 \mathrm{~mL}$ of maternal plasma, a QIAmp Circulating Nucleic Acid Kit (Qiagen, Hilden, Germany) was employed. The extracted cfDNA was eluted in $40 \mu \mathrm{L}$ of AVE buffer (Qiagen). According to the Momguard library preparation protocol, the cfDNA library was prepared and evaluated using a PicoGreen assay and 2100 Bioanalyzer (Agilent Technologies, Palo Alto, CA, USA). Resulting libraries were sequenced using a NextSeq 500 instrument (Illumina Inc., San Diego, CA, USA) or
Ion Torrent (Life Technology, Waltham, MA, USA).

\section{Sequencing data analysis}

Sequencing data were analyzed with an in-house bioinformatics pipeline called GeneBro. In brief, paired-end sequencing reads were binned according to the index sequence and mapped to the reference human genome sequence (hg 19). The tool of Burrows-Wheeler Alignment was used to align the sequences to the human genome, and the uniquely aligned reads were used to calculate the statistical values of euploid pregnancies. By applying an ensemble algorithm to perform a similarity analysis based on features such as fetal fraction, gestational age, or guanine-cytosine (GC) content, test samples were evaluated for the risk of fetal aneuploidy.

\section{Results}

\section{Demographic characteristics}

The Momguard test identified 38 'screen positive' results, including samples that were positive for fetal trisomy ( $T$ ) 21 $(n=31), T 18(n=4)$, and T13 $(n=3)$. The median maternal age of these subjects was 35 years and gestational age was 14.5 weeks (Table 1). When classified by specific aneuploidy, the median maternal ages associated with $\mathrm{T} 13$ cases were lower than those associated with T18 or T21 cases. In contrast, the median gestational ages associated with $\mathrm{T} 13$ cases were the highest of the three at 16.4 weeks. Considering the small number of T13 cases $(n=3)$, this may not be statistically meaningful.

\section{Sequencing and karyotyping results}

Among the 38 'screen positive' results from the Momguard test, 30 cases, including those associated with T21 ( $n=24)$, T18 $(n=3)$, and T13 $(n=3)$ cases, had karyotyping results available during the study period (Table 2). For all T18 and T13 cases,

Table 1. Demographic characteristics of the 38 pregnant women included in the study

\begin{tabular}{ccccc}
\hline Clinical parameter & Classification & Count & Median & Range \\
\hline Maternal age (yr) & All & 38 & 35.0 & $21-42$ \\
& T21 & 31 & 36.0 & $21-42$ \\
& T18 & 4 & 36.5 & $26-40$ \\
& T13 & 3 & 29.0 & $25-31$ \\
\hline \multirow{2}{*}{ Gestational age (wk) } & All & 38 & 14.5 & $11.1-20.7$ \\
& T21 & 31 & 15.1 & $11.1-20.7$ \\
\hline & T18 & 4 & 12.8 & $11.3-13.9$ \\
& T13 & 3 & 16.4 & $11.3-17.7$ \\
\hline
\end{tabular}

T, trisomy. 
Table 2. Confirmatory testing after the Momguard test

\begin{tabular}{|clr|}
\hline Momguard test result & \multicolumn{2}{c}{ Karyotyping result $(\mathrm{n})$} \\
\hline T21 $(n=31)$ & T21 & 23 \\
& Unaffected & 1 \\
\hline T18 $(n=4)$ & In progress & 7 \\
& T18 & 3 \\
\hline T13 $(n=3)$ & Unaffected & 0 \\
\hline & In progress & 1 \\
\hline & T13 & 3 \\
\hline & Unaffected & 0 \\
\hline
\end{tabular}

$\mathrm{T}$, trisomy.

Momguard results were concordant with the karyotyping results. For 121 cases, results from 23 out of 24 cases matched the karyotyping results. Only one case identified as at intermediate risk for aneuploidy by the Momguard test was determined 'unaffected' by karyotyping.

\section{Discussion}

The Momguard test is the first NIPT in Korea developed by original technology called GeneBro. It applies an ensemble algorithm to perform a similarity analysis based on features such as fetal fraction, gestational age, or GC content. The performance of the Momguard test has been assessed in the previous, prospective cohort study carried out in multiple centers, including the Asan Medical Center [16]. The authors showed that the Momguard test is highly accurate in detecting T21 and T18 cases. Due to its rarity, only one T13 sample was identified in that study: it was determined 'intermediate risk' by the Momguard test and was subsequently verified by CVS.

In this study, we attempted to confirm the reliability of the Momguard test with independent samples. We planned to evaluate all 38 'screen positive' results, including those indicating trisomy cases. However, due to time constraints, karyotyping results were not available for all of the samples that tested positive by Momguard. Although the results presented here are limited to a preliminary analysis that verified 30 Momguard results, including those indicating a trisomy, additional results will be available in the near future. Since the evaluation is ongoing, whenever 'screen positive' data becomes available, it will be verified and reported.

Among the 30 cases with both Momguard and karyotyping results, only one case was identified as being at intermediate risk for T21 by the Momguard test. This result was not confirmed by the karyotyping analysis. Since Momguard reports the fetal aneuploidy status as positive (high risk) or negative (low risk) only, the LabGenomics Clinical Lab reported this sample as high risk, even though the result fell in a gray zone. At the same time, the company contacted the pregnant woman who was tested, informed her of the result, and recommended karyotyping to confirm the finding.

In a study by Bianchi et al. [17], the gray zone was defined by samples having a normalized chromosome value within 2.54. For these 'unclassified' samples, including $1.4 \%$ of T21 cases, $1 \%$ of $\mathrm{T} 18$ cases, and $0.4 \%$ of $\mathrm{T} 13$ cases, they did not determine the ploidy status. In the Momguard algorithm, the gray zone was defined by samples in which the ratio of similarity scores for both the center of diploidy and triploidy population in the similarity analysis results is higher than 0.9 . About $0.5 \%$ of all cases fell within this marginal area for determining ploidy status. For these gray zone cases, instead of reporting them as 'unclassified', LabGenomics Clinical Lab followed the strategy outlined above.

Although the Momguard test is a highly reliable screening tool, it may have limitations in identifying some cases that are in the gray zone. To overcome this limitation, further study will be needed to improve the current algorithm. In addition, the performance of this test should be evaluated using a large number of samples from independent cohorts.

Although NIPT by MPS is now accepted as a highly reliable clinical test for detecting fetal aneuploidy, it is still considered a screening test rather than a diagnostic test [18]. Therefore, karyotyping should be recommended to women with positive Momguard results. Since sequencing technologies are rapidly improving and advanced analysis methods continue to be introduced, NIPT is expected to become the first-line of testing for all pregnant women [13]. To better assess the reliability of the Momguard test, further assessments of the Momguard test using a greater number of clinical samples will be needed.

\section{References}

1. Hwang D. The recent trend of prenatal screening. J Genet Med 2008; 5:7-14.

2. Kim S, Kim YH, Min WK. Prenatal serum marker screening in Korea: survey results. Korean J Lab Med 2007;27:28-33.

3. Yi DY, Jung B, Kang MS, Huh JY, Paek JY, Cha DH, et al. The evaluation of integrated test as an antenatal screening test for down's syndrome in Korea. Lab Med Online 2011;1:10-8.

4. Ghaffari SR, Tahmasebpour AR, Jamal A, Hantoushzadeh S, Eslamian 
$\mathrm{L}$, Marsoosi $\mathrm{V}$, et al. First-trimester screening for chromosomal abnormalities by integrated application of nuchal translucency, nasal bone, tricuspid regurgitation and ductus venosus flow combined with maternal serum free $\beta$-hCG and PAPP-A: a 5-year prospective study. Ultrasound Obstet Gynecol 2012;39:528-34.

5. Lau TK, Cheung SW, Lo PS, Pursley AN, Chan MK, Jiang F, et al. Noninvasive prenatal testing for fetal chromosomal abnormalities by low-coverage whole-genome sequencing of maternal plasma DNA: review of 1982 consecutive cases in a single center. Ultrasound Obstet Gynecol 2014;43:254-64.

6. Lo YM, Corbetta N, Chamberlain PF, Rai V, Sargent IL, Redman CW, et al. Presence of fetal DNA in maternal plasma and serum. Lancet 1997;350:485-7.

7. Jensen TJ, Zwiefelhofer T, Tim RC, Džakula Ž, Kim SK, Mazloom $A R$, et al. High-throughput massively parallel sequencing for fetal aneuploidy detection from maternal plasma. PLoS One 2013;8: e57381.

8. Sparks AB, Wang ET, Struble CA, Barrett W, Stokowski R, McBride $C_{\text {, et }}$ al. Selective analysis of cell-free DNA in maternal blood for evaluation of fetal trisomy. Prenat Diagn 2012;32:3-9.

9. Sehnert AJ, Rhees B, Comstock D, de Feo E, Heilek G, Burke J, et al. Optimal detection of fetal chromosomal abnormalities by massively parallel DNA sequencing of cell-free fetal DNA from maternal blood. Clin Chem 2011;57:1042-9.

10. Samango-Sprouse $C$, Banjevic M, Ryan A, Sigurjonsson $S$, Zimmermann $B$, Hill $M$, et al. SNP-based non-invasive prenatal testing detects sex chromosome aneuploidies with high accuracy.
Prenat Diagn 2013;33:643-9.

11. Liao GJ, Gronowski AM, Zhao Z. Non-invasive prenatal testing using cell-free fetal DNA in maternal circulation. Clin Chim Acta 2014;428: 44-50.

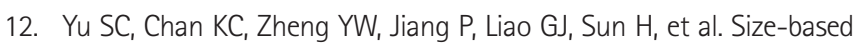
molecular diagnostics using plasma DNA for noninvasive prenatal testing. Proc Natl Acad Sci U S A 2014;111:8583-8.

13. Shea JL, Diamandis EP, Hoffman B, Lo YM, Canick J, van den Boom D. A new era in prenatal diagnosis: the use of cell-free fetal DNA in maternal circulation for detection of chromosomal aneuploidies. Clin Chem 2013;59:1151-9.

14. Yang J. Clinical application of non-invasive prenatal testing using cell free fetal DNA. J Korean Med Assoc 2014;57:771-9.

15. Morain $\mathrm{S}$, Greene MF, Mello MM. A new era in noninvasive prenatal testing. N Engl J Med 2013;369:499-501.

16. Lee MY, Cho DY, Won HS, Hwang AR, Jeong B, Kim J, et al. Performance of Momguard, a new non-invasive prenatal testing protocol developed in Korea. Obstet Gynecol Sci 2015;58:340-5.

17. Bianchi DW, Platt LD, Goldberg JD, Abuhamad AZ, Sehnert AJ, Rava RP; MatErnal BLood IS Source to Accurately diagnose fetal aneuploidy (MELISSA) Study Group. Genome-wide fetal aneuploidy detection by maternal plasma DNA sequencing. Obstet Gynecol 2012;119:890-901.

18. Benn P, Cuckle $H$, Pergament E. Non-invasive prenatal testing for aneuploidy: current status and future prospects. Ultrasound Obstet Gynecol 2013;42:15-33. 